\title{
The volume change due to $F$ centres in alkali halides
}

\author{
Gwendoline Lord $†$ and A M Stoneham \\ Theoretical Physics Division, Atomic Energy Research Establishment, Harwell, Oxfordshire, \\ OX11 ORA
}

Received 11 November 1974

\begin{abstract}
The change in volume due to $F$ centres in alkali halides is calculated using the ion-size correction method of Bartram, Stoneham and Gash. In all cases the volume change $\Delta V$ is positive (expansion of the crystal) and satisfactory agreement with experiment is found in the two cases where measurements are available. The fractional volume change $\left(\Delta V / a^{3}\right)$ decreases systematically with increasing anion or cation size.
\end{abstract}

A number of workers have analysed the volume changes in radiation-damaged alkali halides. These results, when combined with other data involving doped crystals and the interconversion of the various species, permit determination of the contribution to the volume change of $F$ centres. There has been some controversy in the past, for these measurements predict a volume expansion in $\mathrm{KCl}$ and $\mathrm{KBr}$ (see Balzer et al 1966, 1968, 1969, Peisl et al 1966, von Guerard et al 1969, Luty et al 1968, Farnum and Royce 1968) whereas a contraction was predicted by the analysis of spin resonance data for LiF and $\mathrm{KCl}$ (Feuchtwang 1962) and a number of calculations (Kojima 1957, Gourary and Adrian 1957, Wood and Joy 1964). The present calculations, like those of Thommen (1965), agree with the direct measurements in predicting an expansion.

The problem in calculating the volume change for a defect like an $\mathrm{F}$ centre (an electron trapped at an anion vacancy) is that there is cancellation of the major contributions. We shall calculate the various terms separately to demonstrate this.

The volume change caused by a defect can be written simply in terms of the forces $\boldsymbol{F}_{i}$ the defect exerts on the ions at $\boldsymbol{R}_{i}$. If we regard the ions as rigid, ignoring any differences in shell and core coordinates, then the volume change is (eg Temkin 1970):

$$
\Delta V=\frac{\Sigma_{\alpha} \Sigma_{i} R_{i \alpha} F_{i \alpha}}{c_{11}+2 c_{12}}
$$

where $\alpha$ labels cartesian coordinates and the $c_{i j}$ are elastic constants. Note that this expression does not involve the displacements of the ions directly; it is perfectly possible (although not the case here) to have inward motion of the nearest neighbours and overall expansion.

There are three main contributions to the forces on the nearest neighbours (see eg Stoneham and Bartram 1970). One comes from the fact that a negative point charge has

\footnotetext{
$\dagger$ On attachment from St Hugh's College, Oxford.
} 
been replaced by a spread-out distribution which overlaps the adjacent ions. For an s-like function $\psi(r)=R(r)(4 \pi)^{-1 / 2}$ this outward force is

$$
F_{\mathrm{PI}}^{(1)}=\frac{e^{2}}{a^{2}} \int_{a}^{\infty} \mathrm{d} r r^{2} R^{2}(r)
$$

where $a$ is the nearest-neighbour distance. A second, inward, force appears because of the elimination of the repulsive forces between the neighbours and the anion removed in forming the defect. After using the equilibrium condition for the perfect crystal, we find

$$
F_{\text {REP }}^{(1)}=-\frac{e^{2}}{a^{2}} \frac{1}{6} \alpha_{\text {Madelung. }}
$$

Here $\alpha_{\text {Madelung }}$ is 1.7476 , the Madelung constant. Thirdly, there are corrections because the ions are not point charges. In the approximation of Bartram et al (1968) this force can be written:

$$
F_{\mathrm{IS}}^{(1)}=\frac{1}{1-\beta}\left[\beta F_{\mathrm{PI}}^{(1)}+\frac{e^{2}}{a^{2}} B_{+} R^{2}(a)\left(\frac{1}{2}+\frac{2 \sqrt{2}}{3}\right)-\left.\frac{1}{3} C_{+} \frac{\mathrm{d}}{\mathrm{d} r} R^{2}(r)\right|_{a}\right]
$$

where $\beta$ is $(4 \pi)^{-1} \Sigma_{i} B_{i}\left|R\left(\boldsymbol{R}_{i}\right)\right|^{2}$ and the values of $B_{+}$and $C_{+}$are given by Bartram et al. The ion-size force is outward, for it results from a repulsive term in the energy at the cation sites.

Similar contributions arise from the second neighbours, although all are inward. The point-ion term, calculated later, is very small. The repulsive term is not calculated, for the division of repulsive forces between first and second neighbours is not simple. The secondneighbour ion-size term is also small, but is not given in detail. The main effect of the anion ion-size term is in determining the form of the wavefunction.

Table. 1. Volume changes for $F$ centres in alkali halides. The various contributions to

\begin{tabular}{|c|c|c|c|c|c|c|}
\hline \multicolumn{4}{|c|}{ Nearest neighbour } & \multicolumn{2}{|l|}{ Second neighbour } & \multirow[b]{2}{*}{ Experiment } \\
\hline Host & Point ion & Repulsive & Ion size & point ion & Total & \\
\hline $\mathrm{RbF}$ & 0.561 & -0.756 & 1.037 & -0.063 & 0.78 & \\
\hline $\mathrm{KF}$ & 0.528 & -0.765 & $1 \cdot 624$ & -0.054 & $1 \cdot 33$ & \\
\hline $\mathrm{RbCl}$ & $0 \cdot 364$ & -0.603 & $0 \cdot 883$ & -0.032 & 0.61 & \\
\hline $\mathrm{RbBr}$ & 0.444 & -0.735 & 1.096 & -0.039 & 0.77 & \\
\hline $\mathrm{NaF}$ & 0.572 & -0.918 & $2 \cdot 401$ & -0.052 & 2.00 & \\
\hline $\mathrm{KCl}$ & 0.448 & -0.794 & 1.390 & -0.036 & $1 \cdot 01$ & $0.6(a)$ \\
\hline $\mathrm{KBr}$ & 0.426 & -0.736 & $1 \cdot 251$ & -0.035 & 0.91 & $0.6(a),(b),(c),(d) ; 0.8(e)$ \\
\hline $\mathrm{RbI}$ & 0.358 & -0.706 & 0.953 & -0.025 & 0.58 & \\
\hline KI & 0.346 & -0.744 & $1 \cdot 177$ & -0.022 & 0.76 & \\
\hline $\mathrm{NaCl}$ & 0.422 & -0.806 & 1.536 & -0.031 & $1 \cdot 12$ & \\
\hline $\mathrm{LiF}$ & 0.649 & -0.975 & $3 \cdot 130$ & -0.063 & $2 \cdot 74$ & \\
\hline $\mathrm{NaBr}$ & 0.410 & -0.755 & $1 \cdot 325$ & -0.032 & 0.95 & \\
\hline $\mathrm{NaI}$ & 0.340 & -0.728 & $1 \cdot 114$ & -0.021 & 0.71 & \\
\hline $\mathrm{LiCl}$ & 0.523 & -0.936 & 1.980 & -0.041 & 1.53 & \\
\hline $\mathrm{LiBr}$ & 0.587 & -1.002 & 1.890 & -0.050 & 1.43 & \\
\hline LiI & 0.469 & -0.966 & 1.553 & -0.031 & 1.02 & \\
\hline
\end{tabular}
$\Delta V / a^{3}$ are given for the $\mathrm{NaCl}$ structure alkali halides. $\mathrm{CsF}$ is omitted because no elastic data are available, but it is likely that $\Delta V$ is very small indeed.

References: (a) Balzer et al (1968); (b) Peisl et al (1966); (c) Balzer et al (1969); (d) von Guerard et al (1969); (c) Luty et al (1968). 
The results are summarized in table 1, which includes the two known experimental values. It can be seen that expansion of the crystal appears in all cases. However, a contraction would occur if the ion-size forces were ignored. The agreement with experiment is satisfactory where data are available, which is gratifying because of the cancellations involved and because of the very simple wavefunctions used. One point should be mentioned. The original work of Bartram et al assumed that certain factors $\partial U / \partial Q$ did not vary in the lattice relaxation, and so found nearest neighbours moving inwards. This assumption has been removed in the present work and accounts for the expansion found here. One significant trend is seen in the fractional change in volume, $\Delta V / a^{3}$; namely an increase as the size of the ions (both anions and cations) decreases. This is partly a result of systematic trends of the $a^{3}$ denominator with ionic radii.

\section{References}

Balzer R, Peisl H and Waidelich W 1966 Phys. Stat. Solidi 15495

1968 Abstracts of Rome Conference 'Colour Centers in Ionic Crystals' No 12 1969 Phys. Stat. Solidi. 31 K29

Bartram R H, Stoneham A M and Gash P 1968 Phys, Rev. 1761014

Farnum E and Royce B S H 1968 Abstracts of Rome Conference 'Colour Centers in Ionic Crystals' No 59

Feuchtwang T E 1962 Phys. Rev. 1261616

Gourary B S and Adrian F J 1957 Phys. Rev. 1051180

Kojima T 1957 J. Phys. Soc. Japan 12918

Luty F, Costa Ribiero S, Mascarenhas S and Sverzut V 1968 Phys. Rev. 1681080

Peisl H, Balzar R and Waidelich W 1966 Phys. Rev. Lett. 171129

Stoneham A M and Bartram R H 1970 Phys. Rev. B2 3403

Temkin D E 1970 Sov. Phys.-Solid St. 111614

Thommen K 1965 Z. Phys. 186347

von Guerard B, Peisl $\mathrm{H}$ and Waidelich W 1969 Z. Phys. 220

Wood R F and Joy H 1964 Phys. Rev. 136 A451 